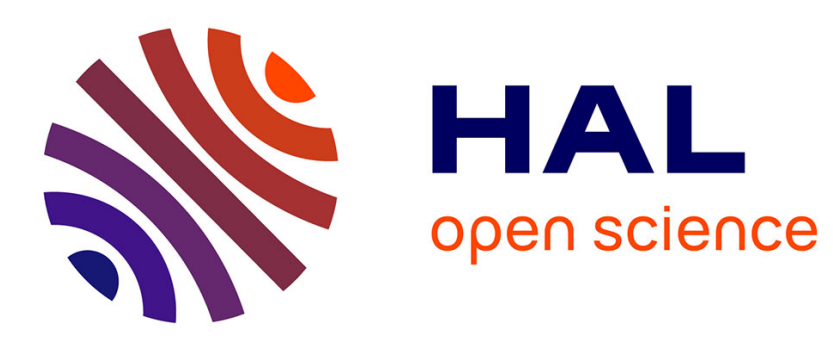

\title{
Fluorescence-enhanced bio-detection platforms obtained through controlled "step-by-step" clustering of silver nanoparticles
}

\author{
Panpan Liu, Ying Zhou, Min Guo, Shuguang Yang, Olivier Félix, David \\ Martel, Yiping Qiu, Ying Ma, Gero Decher
}

\section{To cite this version:}

Panpan Liu, Ying Zhou, Min Guo, Shuguang Yang, Olivier Félix, et al.. Fluorescence-enhanced bio-detection platforms obtained through controlled "step-by-step" clustering of silver nanoparticles. Nanoscale, 2018, 10 (2), pp.848-855. 10.1039/c7nr07486g . hal-03523289

\section{HAL Id: hal-03523289 \\ https://hal.science/hal-03523289}

Submitted on 12 Jan 2022

HAL is a multi-disciplinary open access archive for the deposit and dissemination of scientific research documents, whether they are published or not. The documents may come from teaching and research institutions in France or abroad, or from public or private research centers.
L'archive ouverte pluridisciplinaire HAL, est destinée au dépôt et à la diffusion de documents scientifiques de niveau recherche, publiés ou non, émanant des établissements d'enseignement et de recherche français ou étrangers, des laboratoires publics ou privés. 


\title{
Fluorescence-enhanced bio-detection platforms obtained through controlled "step-by-step" clustering of silver nanoparticles
}

\author{
Panpan Liu, ${ }^{a}$ Ying Zhou, ${ }^{a}$ Min Guo, ${ }^{a}$ Shuguang Yang, ${ }^{b}$ Olivier Félix, ${ }^{c}$ David Martel, ${ }^{c}$ \\ Yiping Qiu, ${ }^{\mathrm{a}, \mathrm{d}}$ Ying Ma *a and Gero Decher*c,e,f \\ ${ }^{a}$ Key Laboratory of Textile Science and Technology of Ministry of Education, \\ College of Textiles, Donghua University, Shanghai, 201620, P. R. China. \\ E-mail: yingma@dhu.edu.cn \\ ${ }^{b}$ State Key Laboratory for Modification of Chemical Fibers and Polymer Materials, \\ College of Material Science and Engineering, Donghua University, Shanghai, 201620, \\ P. R. China \\ 'CNRS Institut Charles Sadron, Strasbourg 67034, France \\ ${ }^{d}$ College of Textiles and Apparel, Quanzhou Normal University, Fujian 362000, \\ P. R. China \\ eFaculté de Chimie, Université de Strasbourg, Strasbourg 67008, France \\ fInternational Center for Frontier Research in Chemistry, Strasbourg 67083, France
}

\begin{abstract}
:
Metal nanoparticle coatings are widely employed as fluorescence-enhanced platforms for highthroughput biological detection; however, complex manufacturing technologies and stringent fabrication procedures hinder their development for use in bioassays. Here, we present the preparation of fluorescencebased bioassay platforms using spray-assisted step-by-step assembly of silver nanoparticles (Ag NPs) and poly(diallyldimethylammonium chloride) (PDDA). This approach allowed us to control the density and the degree of aggregation of Ag NPs on large surfaces which are prerequisites for the development of bioassay platforms with a substantial fluorescence enhancement. After one assembly cycle (1-Ag platform) the adsorbed particles are not forming aggregates or ones composed of very few particles which, as expected, led to poor fluorescence enhancement (1.1) for cyanine 5. Further assembly steps induce the clustering of Ag NPs by multiple electrostatic interactions between PDDA and Ag NPs and thus increase the number of nanoparticles per aggregate in a controlled way. We observed that the nanoparticle island growth takes place first mainly in the plane (2D) and then in the plane and in the third dimension and that the aggregate morphology (2D versus 3D) strongly affects the plasmonic fluorescence enhancement of the fluorescent dye. A substantial fluorescence enhancement (12.3) was measured for a Ag NP platform obtained after twelve assembly cycles. This result is within the ballpark of values reported in the literature for bioassay platforms using metal nanoparticles and opens the route towards the preparation of fluorescence-based bioassay platforms on the large scale.
\end{abstract}

\section{Introduction:}

Fluorescence is one of the most commonly used techniques for the detection of biomolecules and it has been employed extensively in the fields of biotechnology and life sciences. ${ }^{1-4}$ Though fluorescencebased detection is highly sensitive, there is always a demand to push the detection limits. To this end, considerable attention has been given to the modification of the spectral properties of fluorophores and the enhancement of their emissive fluorescence. ${ }^{5-8}$ Two-dimensional (2D) sensing platforms composed of metal nanoparticles (NPs) have frequently been used as fluorescence-enhanced substrates in bio-detection. ${ }^{9-12}$ In such 2D platforms, the metal NPs are considered to play an essential role in fluorescence enhancement, owing to the coupling effect between their surface plasmons and the fluorophores. ${ }^{13,14}$ Their fluorescence enhancement effect is closely related to the interparticle distance ${ }^{15,16}$ and to the fluorophore-nanoparticle distance. ${ }^{17-19}$ The relatively close proximity between the metal NPs enhances the local electric fields around them and thus results in strong fluorescence emission. The fluorescence quenching usually occurs for a fluorophore-nanoparticle distance below 5 $\mathrm{nm}$ because of the nonradiative energy transfer to the metal particle. Beyond $5 \mathrm{~nm}$, the quenching 
effect decreases quickly and a fluorescence enhancement can be observed because of the enhanced excitation and radiative decay pathways by metal nanostructures. It is apparent that the uniform fluorophore-nanoparticle distance on the metal NP platforms is greatly contributing to enhance the fluorophore-nanoparticle coupling efficiency and reduces the number of experimental variables. ${ }^{20,21}$ Therefore, one of the main problems for the construction of these fluorescence-enhanced platforms is the control of the number of particles per aggregate and of their packing.

Up to now, various methods have been employed for the preparation of 2D metal NP platforms, for instance, lithography, ${ }^{22,23}$ physical and chemical vapor deposition, ${ }^{24,25}$ electromigration, ${ }^{26,27}$ template technique, ${ }^{28,29}$ or a combination of these, ${ }^{30,31}$ for use in biological detection. Although these methods worked well for the preparation of metal NP platforms, several drawbacks have hindered their development, such as the tedious preparation procedures, need for high temperatures, and high costs. It is therefore highly desirable to develop simple, cheap and versatile approaches for the construction of metal NP platforms with a satisfactory fluorescence enhancement.

Self-assembly techniques based on non-covalent interactions, which include electrostatic, hydrogen bonding, host-guest, and charge-transfer interactions, are especially attractive as they allow to adjust a large variety of control parameters under mild conditions. ${ }^{32-35}$ It was shown that the control of these non-covalent interactions can induce individual metal NPs in aqueous colloidal suspensions to form aggregates in solution, resulting in fluorescence enhancement. ${ }^{36-39}$ In addition, screening of electrostatic repulsion among metal NPs, achieved by adding polyelectrolytes or inorganic salts to screen the surface charges, has been proven to be effective in obtaining fluorescence enhanced aggregates. ${ }^{40}$ However, approaches using NP colloidal suspensions are not suitable for point-of-care testing due to their inherent stability issues related to the colloidal system. Therefore many groups developed self-assembled nanostructures immobilized on planar substrates.12,41 While record fluorescence enhancement factors up to several thousand times were obtained for single molecules systems, ${ }^{11,41,42}$ fluorescence enhancement factors around several tenfold were observed for selfassembled nanostructures. ${ }^{43,44}$ The substantial value difference between these two systems is explained by the use of various detection modes based on single-molecular level or bulk ensemble measurements. In terms of practical applications platforms based on bulk ensemble measurements compare favourably to single molecule devices due to the ease of their preparation (limited synthetic and technical steps) although their fluorescence enhancement is significantly lower.

Among available self-assembly techniques layer-by-layer ( $L b L$ ) assembly is considered an universal, convenient and efficient method to build nanocomposite films with tunable morphology and functionality. ${ }^{45-53}$ Here we used a variant of LbL assembly in which we intentionally work at a low coverage of NPs per assembly step which strongly favors island growth over the growth of regular dense particle/polyelectrolyte layers.

The objective of the present work is to control the island growth (or step-by-step clustering) in a way so that the resulting NP islands (clusters) have small NP-NP distances for obtaining high fluorescent enhancement. At the same time the step-by-step clustering prevents the formation of large aggregates which would lead to a strong blue shift of the plasmon absorbance. It therefore allows to tailor position and width of the plasmon band well enough for device fabrication.

After synthesis and characterization of Ag NPs we first studied their density, their morphology and their spectroscopic properties on poly(diallyldimethylammonium chloride) (PDDA) terminated multilayers by SEM, UV-vis spectroscopy and fluorescence spectroscopy as a function of spray deposition conditions. In order to demonstrate the importance of the aggregate morphology (2D versus 3D) on spectroscopic properties, we further increased the number of NP per aggregate through step-by-step clustering using PDDA to reduce interparticle distance via charge screening and to grow $\mathrm{NP}$ islands preferentially in 2D prior to 3D. Fluorescence enhancement at low NP density and of NP islands was measured through bioassays using rabbit immunoglobulin $G$ and a conjugate of goat antirabbit immunoglobulin $\mathrm{G}$ with cyanine (Cy5). Cyanine-5 (Cy5), a near-infrared fluorescent dye, has broad applications in biological detection because of its low autofluorescence and limited bleaching. ${ }^{54-}$ ${ }^{56}$ Usually, its fluorescence enhanced factor through the bulk ensemble detection on metal platforms is around $5-20 .{ }^{57-59}$ In our work, the simple and versatile step-by-step assembly allows the controllable 
construction of metal NP platforms, reaching a satisfactory fluorescence enhancement of 12.3-fold after twelve deposition cycles. In addition, the use of spray deposition technique ${ }^{60-62}$ considerably accelerated the assembly process, which is very useful for scaling up.

\section{Experimental: \\ - Materials}

Trisodium citrate dihydrate $\left(\mathrm{Na}_{3} \mathrm{C}_{6} \mathrm{H}_{5} \mathrm{O}_{7} \cdot 2 \mathrm{H}_{2} \mathrm{O}\right)$, poly(sodium 4-styrenesulfonate) (PSS, average $\mathrm{Mw}=70$ 000 ) and poly(diallydimenthylammonium chloride) (PDDA, average $\mathrm{Mw}=100$ 000-200 000, $20 \mathrm{wt} \%$ aqueous solution) were purchased from Sigma-Aldrich. Silver nitrate $\left(\mathrm{AgNO}_{3}\right)$ was obtained from $\mathrm{J} \& \mathrm{~K}$ Chemical Co., Ltd. Goat anti-rabbit IgG-Cy5 and rabbit IgG used in bioassay experiments was provided by Thermo Fisher Scientific. All chemicals were used as received without further purification. Milli- $Q$ water $(18.2 \mathrm{M} \Omega . \mathrm{cm})$ was used throughout the present study. Spray guns (SATAminijet 3000B HVLP) were purchased from SATA GmbH\&Co.KG (Germany).

\section{- Synthesis of Ag NPs}

The silver nanoparticles (Ag NPs) used for platform preparation were synthesized by the citrate reduction of silver nitrate using a procedure previously described in the literature. ${ }^{63}$ Briefly, $90 \mathrm{mg}$ $\mathrm{AgNO}_{3}$ were dissolved in $500 \mathrm{~mL}$ of Milli-Q water and the resultant solution was heated to boiling under stirring. Then, $10 \mathrm{~mL}$ of $1 \%$ sodium citrate were added. After boiling for $1 \mathrm{~h}, \mathrm{Ag}$ NPs were obtained. The concentration of the resulting Ag NP colloidal suspension was calculated to be $1.42 \times 10^{-10} \mathrm{M}$, as calculated from the absorbance of the 6-fold diluted colloidal suspension (Fig. 1c) and the already reported extinction coefficient for $60 \mathrm{~nm} \mathrm{Ag} \mathrm{NPs}\left(6.75 \times 1010 \mathrm{M}^{-1} \mathrm{~cm}^{-1}\right) \cdot{ }^{64}$ During the reaction, the color of the colloidal suspension changed from transparent to opaque yellow-gray. The as-prepared $\mathrm{Ag}$ NPs are negatively charged as confirmed by zeta potential measurements $(-30 \mathrm{mV})$. Under stirring, the Ag NP colloidal suspensions can be kept over 3 days at $4{ }^{\circ} \mathrm{C}$.

\section{- Step-by-step clustering of Ag NPs to prepare Ag platforms}

Cleaned quartz slides were modified with a PDDA/(PSS/PDDA) $\times 3$ film using alternate dipping into PDDA ( $1 \mathrm{mg} \mathrm{mL}^{-1}, 15 \mathrm{~min}$ ) and PSS ( $1 \mathrm{mg} \mathrm{mL}^{-1}, 15 \mathrm{~min}$ ) with intermediate rinsing ( $1 \mathrm{~min}, 3$ times) and drying steps. Then, the step-by-step clustering of Ag NPs was conducted on the pretreated quartz substrates by spray-assisted assembly as follows. Firstly, the as-prepared Ag NP aqueous colloidal suspension was sprayed on the pretreated quartz at 20 psi for $30 \mathrm{~s}$, followed by the rinsing with Milli$Q$ water to remove physical absorption. Subsequently, $1 \mathrm{mg} \mathrm{mL}^{-1}$ PDDA aqueous solution was sprayed at $30 \mathrm{psi}$ for $10 \mathrm{~s}$ and adsorbed on Ag NPs. The spraying and rinsing steps were repeated until reaching the desired Ag platform ending with an Ag NP layer. No drying step was used during assembly.

\section{- Bioassay procedures}

The substrates uncoated and coated by Ag platforms were covered with $150 \mu \mathrm{L}$ of $17 \mu \mathrm{g} \mathrm{mL}^{-1} \operatorname{lgG}$ aqueous solution with a pH of 7.4 and then incubated in a shaker $(90 \mathrm{rpm})$ at $20^{\circ} \mathrm{C}$ for $1 \mathrm{~h}$. Then, the slides were immersed into $150 \mu \mathrm{L}$ of $1 \%$ bovine serum albumin (BSA) for $1 \mathrm{~h}$, followed by $150 \mu \mathrm{L}$ of goat anti-rabbit IgG-Cy5 at $17 \mu \mathrm{g} \mathrm{mL}^{-1}$ for $1 \mathrm{~h}$. BSA was used to block the substrate sites during the adsorption of goat antirabbit IgG-Cy5 on Ag platform. BSA molecules covered completely the $\mathrm{Ag}$ platform, including the surface of the PDDA/(PSS/ PDDA) $\times 3$ and the Ag NPs. Subsequently, the goat anti-rabbit IgG-Cy5 replaced the BSA on Ag NPs to interact with the exposed IgG, owing to their specific binding. All samples were rinsed with a PBS solution $(\mathrm{pH}=7.4)$ for three times after each step, and were always kept in a wet condition to prevent protein unfolding or denaturation.

\section{- Finite-difference time-domain (FDTD) calculations}

The FDTD method was employed to determine the electric field intensities and distributions at the surface of planar and stacked silver cluster. In the simulation, Ag NPs were defined as spheres with a diameter of $68 \mathrm{~nm}$. The planar clusters were simply modeled as three Ag NPs arranged linearly and 
horizontally. And the stacked clusters were modeled as three Ag NPs on the bottom and two Ag NPs on the top. All interparticle distances were set to $1 \mathrm{~nm}$. Irradiation was carried out using a $633 \mathrm{~nm}$ plane light wave normal to the samples. As boundary conditions, all outmost sides were defined as perfect matching layer (PML). ${ }^{39}$

\section{- Characterization}

UV-Vis spectra were measured using a Hitach U-3030 UV-Vis spectrometer. The TEM image of Ag NPs was taken using a JEM-2100F transmission electron microscope (JEOL, Japan). The surface morphologies of Ag platforms were determined by a Hitachi S- 4800 scanning electron microscope. Fluorescence spectra and lifetime were recorded using a Quant Master 40 and Timemaster 3 fluorescence spectrometer (Photon Technology International Inc.) under an excitation of $615 \mathrm{~nm}$, respectively. Fluorescence intensity images were captured on a TCS SP5 confocal laser scanning microscope (Leica company, Germany) with an excitation at $633 \mathrm{~nm}$.

\section{Results and discussion:}

Here we report on the control of particle clustering for providing a maximum of fluorescence enhancement using an easy, cheap and scalable approach for preparing new sensors coatings for future point-of-care testing. Ag NPs were chosen as plasmonic nanostructures and were prepared by conventional citrate reduction (see Experimental section for details). ${ }^{63}$ As shown in Fig. 1a, the asobtained Ag NPs were a mixture of spherical, ellipsoidal and rod-like particles. To determine the diameter and size distribution of the resulting Au NPs, both the major and minor axes of more than 200 NPs were measured. The average diameter was determined to be $68 \pm 9 \mathrm{~nm}$ (Fig. 1b), which meets the size requirement of Ag NPs for fluorescence enhancement $(>40 \mathrm{~nm}) .65$ The citrate ligands on the Ag NPs lead to a surface potential of $-30 \mathrm{mV}$. This negative charge stabilized their colloidal suspensions. The UV-Vis absorption spectrum of the Ag NP aqueous colloidal suspension (Fig. 1c) shows a broad and asymmetric peak which can be attributed to the convoluted signal of the dipole and quadrupole plasmon resonances of individual Ag NPs at 417 and $388 \mathrm{~nm} .66$ Macroscopically the colloidal suspensions have an opaque yellow-gray appearance, as shown in the inset of Fig. 1c, which corresponds well to the expectations for the size and concentration range of the colloidal particle suspensions reported here.

(a)

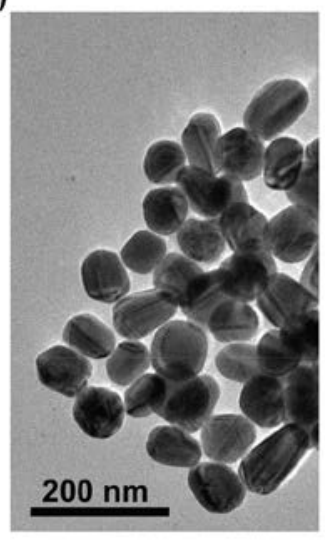

(b)
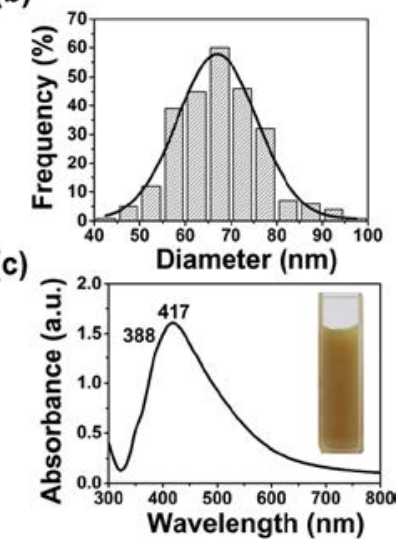

Fig. 1 (a) TEM image of the Ag NPs used for platform preparation and (b) histogram showing an average diameter of $68 \pm 9$ $\mathrm{nm}$ with a wide size distribution. (c) UV-Vis absorption spectrum and photograph (inset) of the Ag NP aqueous suspension.

The Ag NPs were deposited on the substrate by spray-assisted assembly (Fig. 2a). The quartz substrate was precoated with a multilayer film composed of PDDA and poly (sodium 4-styrenesulfonate) (PSS), which is denoted as PDDA/(PSS/PDDA) $\times 3$, to provide sufficient positive charges for the adsorption of the negatively charged Ag NPs. Subsequently, the aqueous colloidal suspension of Ag NPs was sprayed perpendicular to the receiving surface, with a fixed distance of $15 \mathrm{~cm}$ between the nozzle and the substrate. All spraying steps were carried out at identical conditions. Fig. $2 \mathrm{~b}$ shows the Ag platform 
obtained using a pressure of $20 \mathrm{psi}$ for $30 \mathrm{~s}$ after a one-time spraying. The Ag NPs were distributed randomly on the substrate and were fairly isolated from each other, owing to their electrostatic repulsion which is weakly screened by polyelectrolytes present on the surface. ${ }^{67}$ To assess the fluorescence enhancement, the average interparticle distances (L) of the one-time sprayed $\mathrm{Ag}$ platform were calculated as

$$
L=\frac{a}{\sqrt{n}}-D
$$

where $a$ is the length of the square deposition area, and $D$ is the average diameter of the deposited $A g$ NPs $(68 \mathrm{~nm})$, and $\mathrm{n}$ is the number of Ag NPs deposited on the object area counted using the software package Image J. For the Ag platform in Fig. $2 \mathrm{~b}$ (obtained by one-time spraying at 20 psi for $30 \mathrm{~s}$ ), the average interparticle distance was determined to be $222 \mathrm{~nm}$. This value is much higher than the radius of the used Ag NPs (34 nm), which is the maximum interparticle distance (LMax) for strong fluorescence enhancement. ${ }^{9,68}$ Of course, the fluorescence enhancement of this one-time sprayed Ag platform is expected to be poor. As shown in Fig. 2c, our Ag platform displayed similar fluorescence intensity at $685 \mathrm{~nm}$ with the PDDA/(PSS/PDDA) $\times 3$ modified quartz control sample when being treated with rabbit immunoglobulin $\mathrm{G}(\mathrm{IgG})$ and goat anti-rabbit immunoglobulin $\mathrm{G}$ labeled with cyanine-5 (denoted as goat anti-rabbit IgG-Cy5). Thus, the fluorescence enhancement factor (EF) is only 1.1.

(a)

(b)

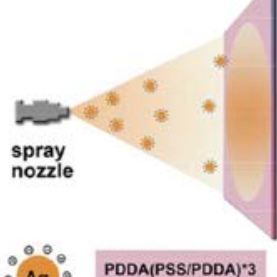

(d)
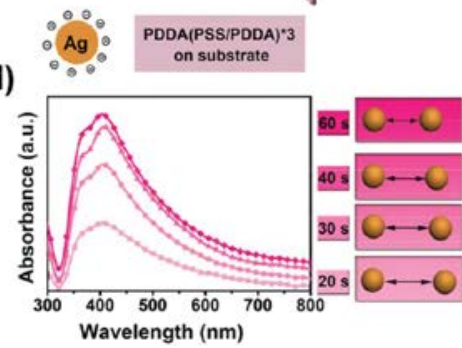

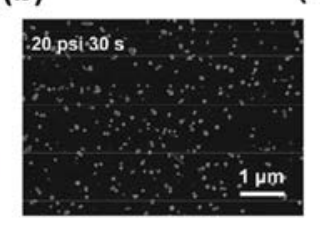

(e) (c)
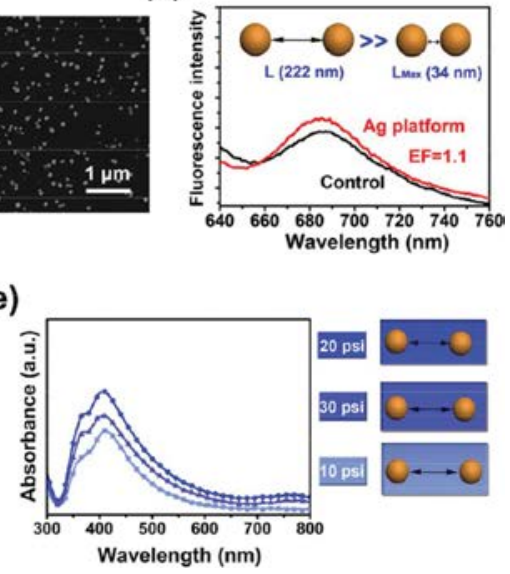

Fig. 2 (a) Schematic of spraying Ag NPs on the substrate. (b) SEM image of a one-time sprayed Ag platform at 20 psi for 30 s. (c) Fluorescence intensity of goat anti-rabbit IgG-Cy5 dried onto the one-time sprayed Ag platform (20 psi, $30 \mathrm{~s})$ and the bare quartz modified with PDDA/(PSS/PDDA) $\times 3$ (the control). UV-Vis absorption spectra and sketches of average interparticle distance (L) for one-time sprayed Ag platforms (d) at 20 psi for 20, 30, 40, 60 s and (e) at 10, 20, and 30 psi for 30 s.

In order to improve the fluorescence enhancement of onetime sprayed Ag platforms, the spraying time and the operating pressure were varied for reducing the interparticle distance. The UV-Vis absorption spectra of the one-time sprayed Ag platforms were recorded to quantify the Ag NPs deposited on the substrate as a function of the spraying time (Fig. 2d) Compared with those of the aqueous colloidal suspension of Ag NPs, the plasmon resonance peaks of the one-time sprayed Ag platforms were blueshifted to 404 and $372 \mathrm{~nm}$, as a result of changing the medium around the Ag NPs from water to air or PDDA layer. ${ }^{69}$ The polyelectrolyte induced blue-shift of the plasmon band of metal nanoparticles that has been reported before. ${ }^{70}$ More importantly, with the augmentation of spraying time from 20 to 30 , 40 and $60 \mathrm{~s}$, the absorbance increased towards a maximum value indicating that the increasing NP adsorption is reaching a plateau at prolonged spraying times. The increasing density of Ag NPs was easily visualized by scanning electron microscopy (SEM; see ESI Fig. S1+). Based on the SEM images, the average interparticle distances (L) after spray times of 20,30, 40 and $60 \mathrm{~s}$ were calculated to be 292, 222, 215 and $181 \mathrm{~nm}$, respectively, as illustrated in the right-hand panel of Fig. 2d. For a constant spray time of $30 \mathrm{~s}$, the adsorption of Ag NPs did not continuously increase when the spray pressure 
was increased from 10 to 20 and 30 psi, but instead reached a maximum at 20 psi (Fig. 2e). The arrival of spray droplets on the receiving surface is a complex process, increasing the spray pressure leads to higher mass transport, but may also remove weakly bound material. As one-time assembly process led to a low density of mostly individual NPs it was necessary to increase the number of NPs per aggregate to meet the requirement of Ag NP platforms in fluorescence enhancement.

Ag NPs were then step-by-step assembled with the counter polyelectrolyte PDDA to yield bio-detection platforms with a substantial fluorescence enhancement. At our optimal spraying conditions ( $20 \mathrm{psi}$ for $30 \mathrm{~s})$, the step-by-step assembled Ag platforms were prepared conveniently and rapidly on PDDA/

(PSS/PDDA) $\times 3$ pretreated quartz substrates (see Experimental section for details) through the electrostatic interactions between the negatively charged Ag NPs and the positively charged PDDA. In all films, Ag NPs were deposited as last step. Fig. 3a shows the UV-Vis absorption spectra of the resulting assembled Ag platforms. With the assembly of Ag NPs, the characteristic peak of the dipole plasmon resonance increased linearly (Fig. 3b) and was blue-shifted from 404 to $381 \mathrm{~nm}$ owing to the increase of electron delocalization ${ }^{71}$ among the Ag NPs. Furthermore, we noted the appearance of a broad peak at around $700 \mathrm{~nm}$, which is associated with the in-plane resonance of Ag NPs and strongly depends on the interparticle distances.65 The surface morphologies of Ag platforms with 5, 12 and 20 assembly cycles (denoted as 5-Ag, 12-Ag and 20-Ag platforms, respectively) were characterized by SEM (Fig. 3c). Further assembly steps of NPs on 1-Ag platform led to islands growth which starts in-plane (planar clusters) and later also extends in the third dimension (stacked clusters). The Ag NPs are initially deposited on the substrate with fairly large gaps between them (Fig. 2b). During the subsequent stepby-step clustering (Fig. 3c), the additionally deposited particles are more and more filling the interspaces likely facilitated by the electrostatic screening of the repulsion between the negatively charged particles and by the electrostatic attraction of the particles by the cationic PDDA present on the surface. When the interspaces were not big enough, stacked structures of Ag NPs began to appear on the surface. In any case, the step-by-step clustering enables the construction of Ag platforms with successively smaller interparticle distances, which contributes to the enhancement of the electric field around the Ag NPs. In order to estimate the fluorescence enhanced capability of the step-by-step assembled Ag platforms, computations of the electric field density for the Ag clusters were conducted using the finite-difference time-domain (FDTD) method. ${ }^{39,72,73}$ Fig. $3 d$ shows the electric field distribution of the planar and stacked Ag clusters under perpendicular irradiation with a $633 \mathrm{~nm}$ plane light wave. The interparticle distance was set to $1 \mathrm{~nm}$, because the Ag NPs are concatenated by the polyelectrolyte PDDA on the nanoscale. As shown in the corresponding line plot (Fig. 3e), the electromagnetic field amplitudes along the lines of $a-a^{\prime}, b-b^{\prime}$ and $c-c^{\prime}$ in Fig. $3 d$ of the planar and stacked $\mathrm{Ag}$ clusters were presented. The electric field intensity at the center point between two adjacent NPs has maximum values of 112.7 (planar) and 57.3 (stacked), respectively. This result indicates the negative effect of the stacking structure on the electric field enhancement. Moreover, the electric field of a planar cluster composed of 5 aggregated Ag NPs was also simulated by FDTD (Fig. S3 of ESI + ). In comparison with the stacked structure in Fig. 3d, the 5-aggregated Ag NPs in the planar structure showed a higher electric field intensity (93.6), underlining the importance of 2D nanostructures in the electric field enhancement and fluorescence enhancement.

To characterize the fluorescence enhancement, we measured the fluorescence of goat anti-rabbit IgGCy5 on IgG modified Ag platforms after 3, 5, 8, 10, 12, 15 and 20 assembly cycles and on a quartz slide coated only with a PDDA/(PSS/PDDA) $\times 3$ film (control). As shown in Fig. 4a, the emission region of Cy5 is centered at $\sim 685 \mathrm{~nm}$, which overlaps with the emerging broad absorption peak ( $700 \mathrm{~nm}$ ) of the step-by-step assembled Ag platforms. This wavelength overlap is expected to facilitate fluorescence enhancement. ${ }^{74,75}$ The large goat anti-rabbit IgG molecules, which are several nanometers in size, ${ }^{10,76}$ may also help to separate the fluorescent $\mathrm{Cy} 5$ dye molecules from the plasmonic Ag NPs for reducing fluorescence quenching effects.77 Integration of these fluorescence intensities gave enhancement factors for the step-by-step assembled Ag platforms as compared with the control (PDDA/ (PSS/PDDA) $\times 3$ on quartz). In Fig. 4b, with the assembly of Ag NPs, these enhancement factors initially increased to a maximum of 3.1 for the $12-\mathrm{Ag}$ platform, followed by a decrease. The above-mentioned negative 
effect of the stacking structure on the electric field density was believed to be responsible for this decreased enhancement factor after 12 assembly cycles. Additionally, we attributed the rebound of

(a)

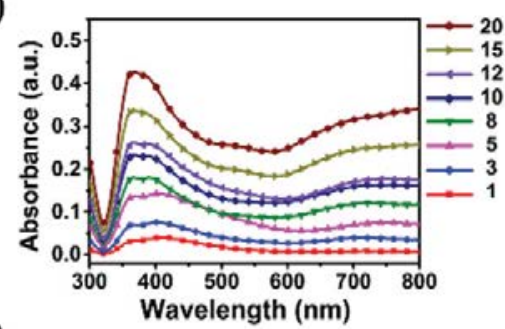

(b)

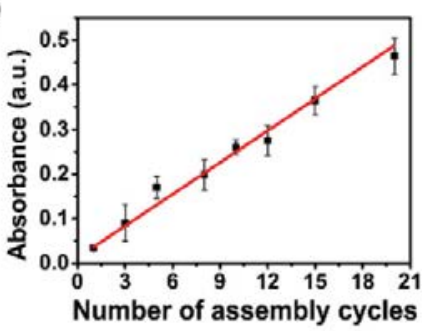

(c)

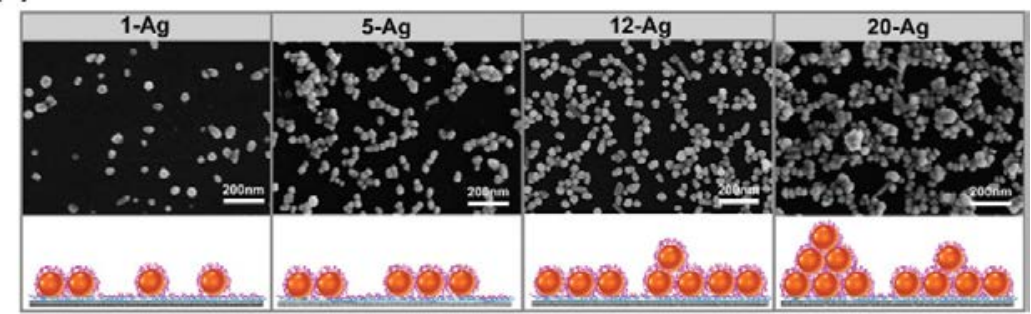

(d)

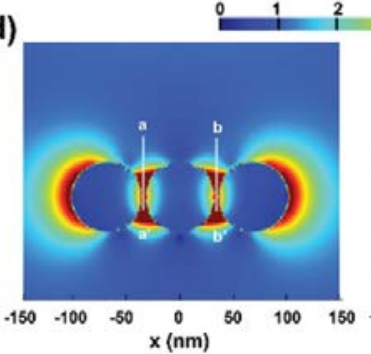

(e)

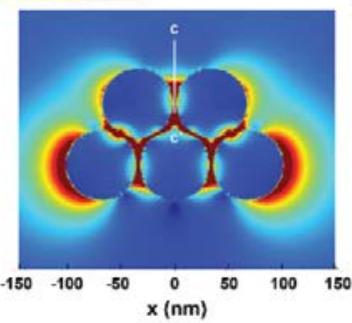

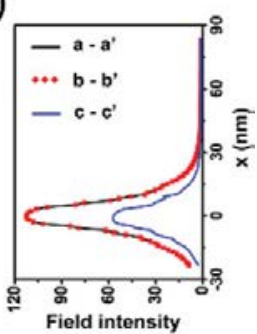

Fig. 3 (a) UV-Vis absorption spectra and (b) absorption maxima around $404 \mathrm{~nm}$ of step-by-step assembled Ag platforms with different assembly cycles. (c) SEM images of the 5-Ag, 12-Ag and 20-Ag platforms. The insets illustrate the step-by step clustering of Ag NPs. (d) Electric field density distribution and (e) corresponding line plot of field enhancement in the gaps between two particles for the aggregated Ag NPs with the planar and stacked structures simulated using FDTD.

the enhancement factor for the 20-Ag platform (i.e., its rise compared with that of the 15-Ag platform) to the coincidence of the stacking and filling of Ag NPs during assembly. This shows that the fluorescence enhancement of metal nanomaterial platforms can be fine-tuned by controlling the stepby-step process. Furthermore, the fluorescence enhancement is generally accompanied by a decrease in the fluorescence lifetime, both of which are derived from the high radiative decay rate of the fluorophores. ${ }^{13,31}$ In Fig. 4c, the fluorescence intensity decay of anti-rabbit IgG-Cy5 shows a shorter Cy5 lifetime of $0.94 \mathrm{~ns}$ for the 12-Ag platform, compared with that of the control (1.36 ns). The 31\% lifetime shortening suggests that the enhanced fluorescence intensity of the 12-Ag platform mainly comes from the intensive electric field rather the increased radiative decay rate. This conclusion also could be drawn from the weak absorption of 12-Ag platform at the emission peak of Cy5 ( 685 nm). The enhanced fluorescence on the 12-Ag platform was readily observed in photographs taken using a confocal laser scanning microscope (CLSM) (Fig. 4d). Compared with the control sample (bottom half), Cy5 on the 12-Ag platform (upper half) exhibited a brighter red color. The corresponding enhancement factor of the 12-Ag platform was calculated to be 3.0 from the analysis of the color intensity by Image J. This result is in agreement with the value calculated from the fluorescence emission spectra (3.1). To reveal the potential of the $12-\mathrm{Ag}$ platform on biological detection, rabbit IgG was adsorbed onto the Ag nanostructure by physisorption,76 followed by the intermediate deposition of bovine serum albumin (BSA) prior to the deposition of goat anti-rabbit IgG-Cy5. The details are described in Experimental section. Concentrating the local electric field around the planar Ag clusters, in which the Ag NPs are in relatively close proximity because of the electrostatic attraction with PDDA, accelerated the excitation of $\mathrm{Cy} 5$ and thus enhanced the fluorescence. The fluorescence enhancement on the 12Ag platform over the control, a quartz slide coated with a PDDA/(PSS/PDDA) $\times 3$ film, was determined 
from the fluorescence emission images in Fig. 5b. The average fluorescence intensity for Cy5 was 2.1 \pm 1.1 for the control and $25.8 \pm 7.1$ for the 12 -Ag platform, respectively. The satisfactory enhancement factor of 12.3 indicates that the controlled step-by-step clustering of metal NPs allows the simple and fast preparation of efficient fluorescence enhancing platforms for bio-detection.

(a)

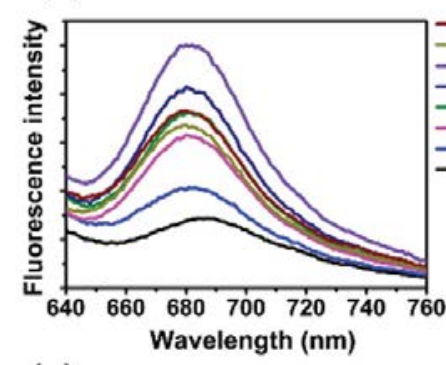

(c)

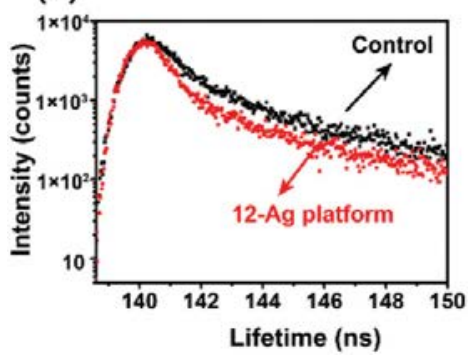

(b)

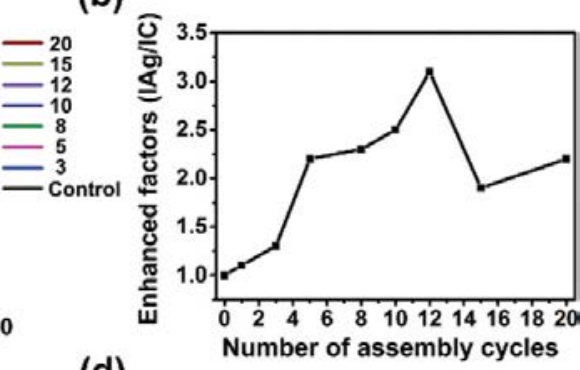

(d)

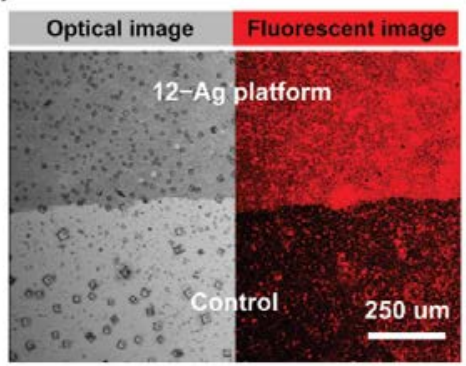

Fig. 4 (a) Fluorescence intensity of goat anti-rabbit IgG-Cy5 dried onto step-by-step assembled Ag platforms with different assembly cycles. (b) Fluorescence enhancement factors of step-by-step assembled Ag platforms as a function of the number of assembly cycles, compared to the control of a bare quartz modified with PDDA/(PSS/PDDA) $\times 3$. (c) Fluorescence lifetimes and (d) CLSM images of goat anti-rabbit IgG-Cy5 on the 12-Ag platforms and on the control.

\section{Conclusions.}

In summary, we have shown that step-by-step clustering is a simple, cheap and versatile method for the preparation of self-assembled metal NP-based platforms with controlled aggregate density and morphology. This approach based on a low coverage of NPs par assembly step favours island growth over the growth of regular dense particle/polyelectrolyte layer as reported for LbL-assembled systems.

(a)

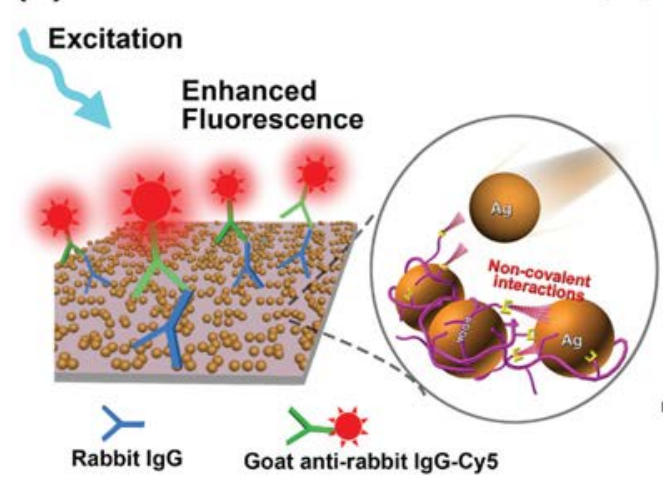

(b)

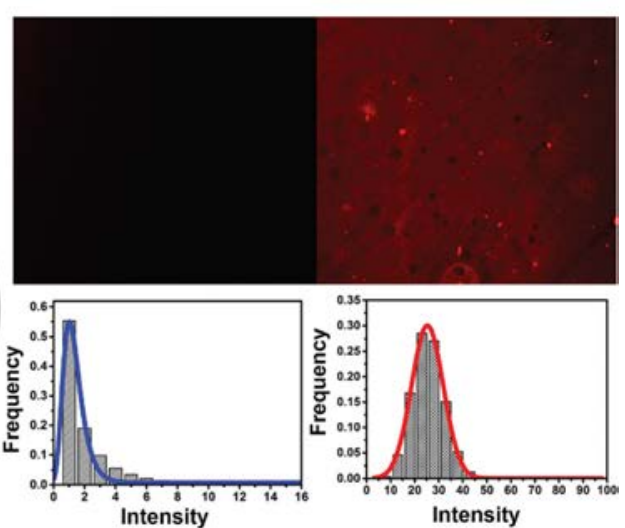

Fig. 5 (a) Schematic illustration of the bioassay procedure on step-by-step assembled Ag platforms. (b) CLSM images and fluorescence intensity distribution of $\mathrm{Cy} 5$ on the control and on the 12-Ag platform.

Starting from mostly individual NPs, we showed that this process can be tuned to lead to an island growth that starts in-plane and later also extends in the third dimension. So the resulting NP islands (clusters) have small NP-NP distances for obtaining high fluorescent enhancement. While a poor fluorescence enhancement (1.1) was observed at low NP density, a substantial fluorescence 
enhancement (12.3) was measured for an Ag NP platform obtained after twelve deposition cycles. This fluorescence enhancement value is within the ballpark of values reported in the literature for biossay platforms using metal nanoparticles. Due to the ease of controlling the position of the plasmon band for tuning the fluorescence enhancement through aggregate size and NP-NP distance, step-by-step clustering fulfils two important prerequisites for mass-scale device fabrication.

\section{Acknowledgements}

This work was supported by National Key Research and Development Program of China (No. 2016YFB0303300), and National Natural Science Foundation of China (No. 21304016).

\section{Notes and references}

1 B. N. Giepmans, S. R. Adams, M. H. Ellisman and R. Y. Tsien, Science, 2006, 312, 217-224.

2 T. Schlichthaerle, M. T. Strauss, F. Schueder, J. B. Woehrstein and R. Jungmann, Curr. Opin. Biotechnol., 2016, 39, 41-47.

3 X. Michalet, F. F. Pinaud, L. A. Bentolila, J. M. Tsay, S. Doose, J. J. Li, G. Sundaresan, A. M. Wu, S. S. Gambhir and S. Weiss, Science, 2005, 307, 538-544.

4 E. M. Carstea, J. Bridgeman, A. Baker and D. M. Reynolds, Water Res., 2016, 95, 205-219.

5 K. Aslan and C. D. Geddes, Plasmonics, 2008, 3, 89-101.

6 E. M. Goldys and F. Xie, Sensors, 2008, 8, 886-896.

7 S. Lin, H. Sharma and M. Khine, Adv. Opt. Mater., 2013, 1, 568-572.

8 Y.-H. Chan, J. Chen, S. E. Wark, S. L. Skiles, D. H. Son and J. D. Batteas, ACS Nano, 2009, 3, 1735-1744.

9 J. J. Giner-Casares and L. M. Liz-Marzán, Nano Today, 2014, 9, 365-377.

10 S. M. Tabakman, L. Lau, J. T. Robinson, J. Price, S. P. Sherlock, H. Wang, B. Zhang, Z. Chen, S. Tangsombatvisit, J. A. Jarrell, P. J. Utz and H. Dai, Nat. Commun., 2011, 2, 466.

11 A. Kinkhabwala, Z. Yu, S. Fan, Y. Avlasevich, K. Müllen and W. E. Moerner, Nat. Photonics, 2009, 3, 654-657.

12 A. Camposeo, L. Persano, R. Manco, Y. Wang, P. Del Carro, C. Zhang, Z.-Y. Li, D. Pisignano and Y. Xia, ACS Nano, 2015, 9, 10047-10054.

13 C. D. Geddes and J. R. Lakowicz, J. Fluoresc., 2002, 12, 121-129.

14 P. P. Pompa, L. Martiradonna, A. Della Torre, F. Della Sala, L. Manna, M. De Vittorio, F. Calabi, R. Cingolani and R. Rinaldi, Nat. Nanotechnol., 2006, 1, 126-130.

$15 \mathrm{~J}$. Leem, M. C. Wang, P. Kang and S. Nam, Nano Lett., 2015, 15, 7684-7690.

16 R. M. Bakker, V. P. Drachev, Z. Liu, H.-K. Yuan, R. H. Pedersen, A. Boltasseva, J. Chen, J. Irudayaraj, A. V. Kildishev and V. M. Shalaev, New J. Phys., 2008, 10, 125022.

17 P. Anger, P. Bharadwaj and L. Novotny, Phys. Rev. Lett., 2006, 96, 113002.

18 J. R. Lakowicz, Anal. Biochem., 2001, 298, 1-24.

19 C. Tserkezis, N. Stefanou, M. Wubs and N. A. Mortensen, Nanoscale, 2016, 8, 17532.

20 N. Akbay, J. R. Lakowicz and K. Ray, J. Phys. Chem. C, 2012, 116, 10766-10773.

21 X. Wang, S. Li, P. Zhang, F. Lv, L. Liu, L. Li and S. Wang, Adv. Mater., 2015, 27, 6040-6045.

22 T. Lohmueller, L. Iversen, M. Schmidt, C. Rhodes, H. L. Tu, W. C. Lin and J. T. Groves, Nano Lett., 2012, 12, 1717-1721.

23 A. Cerf, G. Molnar and C. Vieu, ACS Appl. Mater. Interfaces, 2009, 1, 2544-2550.

24 Y. Zhang, A. Padhyay, J. E. Sevilleja, R. L. Guerrant and C. D. Geddes, J. Phys. Chem. C, 2010, 114, 7575-7581.

25 K. Sugawa, T. Tamura, H. Tahara, D. Yamaguchi, T. Akiyama, J. Otsuki, Y. Kusaka, N. Fukuda and H. Ushijima, ACS Nano, 2013, 7, 9997-10010.

26 B. Yang, N. Lu, D. Qi, R. Ma, Q. Wu, J. Hao, X. Liu, Y. Mu, V. Reboud, N. Kehagias, C. M. Torres, F. Y. Boey, X. Chen and L. Chi, Small, 2010, 6, 1038-1043.

27 L. Shang, H. Chen and S. Dong, J. Phys. Chem. C, 2007, 111, 10780-10784.

28 D. Wang, A. Yang, A. J. Hryn, G. C. Schatz and T. W. Odom, ACS Photonics, 2015, 2, 1789-1794. 
29 Q. Fu, Z. Zhan, J. Dou, X. Zheng, R. Xu, M. Wu and Y. Lei, ACS Appl. Mater. Interfaces, 2015, 7, 133223328.

30 S. Y. Cho, H. J. Jeon, H. W. Yoo, K. M. Cho, W. B. Jung, J. S. Kim and H. T. Jung, Nano Lett., 2015, 15, $7273-7280$.

31 C. L. Wirth, M. De Volder and J. Vermant, Langmuir, 2015, 31, 1632-1640.

32 J. M. Lehn, Angew. Chem., Int. Ed. Engl., 1988, 27, 89-112.

33 K. Tashiro and T. Aida, Chem. Soc. Rev., 2007, 36, 189-197.

34 J. Hirschberg, L. Brunsveld, A. Ramzi, J. Vekemans, R. P. Sijbesma and E. W. Meijer, Nature, 2000, 407, 167-170.

35 S. I. Stupp, V. LeBonheur, K. Walker, L. S. Li, K. E. Huggins, M. Keser and A. Amstutz, Science, 1997, 276, 384-389.

36 R. Gill, L. Tian, W. R. C. Somerville, E. C. Le Ru, H. van Amerongen and V. Subramaniam, J. Phys. Chem. C, 2012, 116, 16687-16693.

37 J.-W. Liaw, J.-H. Chen, C.-S. Chen and M.-K. Kuo, Opt. Express, 2009, 17, 13532-13540.

38 G. Chen, Y. Wang, M. Yang, J. Xu, S. J. Goh, M. Pan and H. Chen, J. Am. Chem. Soc., 2010, 132, 3644645.

39 Z. Jian, F. Yi, M. H. Chowdhury and J. R. Lakowicz, Nano Lett., 2007, 7, 2101-2107.

40 I. O. Osorio-Roman, A. R. Guerrero, P. Albella and R. F. Aroca, Anal. Chem., 2014, 86, 10246-10251.

41 T. Zhang, N. Gao, S. Li, M. J. Lang and Q. H. Xu, J. Phys. Chem. Lett., 2015, 6, 2043-2049.

42 H. Yuan, S. Khatua, P. Zijlstra, M. Yorulmaz and M. Orrit, Angew. Chem., Int. Ed., 2013, 52, 12171221.

43 B. Yang, N. Lu, D. Qi, R. Ma, Q. Wu, J. Hao, X. Liu, Y. Mu, V. Reboud, N. Kehagias, C. M. Torres, F. Y. Boey, X. Chen and L. Chi, Small, 2010, 6, 1038-1043.

44 C. Wang, W. Zhu, Y. Lan, M. Zhang, T. Tian, H. Wang and G. Li, J. Phys. Chem. C, 2014, 118, 1075410763.

45 G. Decher, Science, 1997, 277, 1232-1237.

46 H. Lee, M. L. Alcaraz, M. F. Rubner and R. E. Cohen, ACS Nano, 2013, 7, 2172-2185.

47 D. G. Shchukin, M. Zheludkevich, K. Yasakau, S. Lamaka, M. G. S. Ferreira and H. Möhwald, Adv. Mater., 2006, 18, 1672-1678.

48 Z. Tang, Y. Wang, P. Podsiadlo and N. A. Kotov, Adv. Mater., 2006, 18, 3203-3224.

49 X. Zhang, H. Chen and H. Zhang, Chem. Commun., 2007, 1395-1405.

50 S. A. Castleberry, W. Li, D. Deng, S. Mayner and P. T. Hammond, ACS Nano, 2014, 8, 6580-6589.

51 E. Kharlampieva, V. Kozlovskaya, B. Wallet, V. V. Shevchenko, R. R. Naik, R. Vaia, D. L. Kaplan and V. V. Tsukruk, ACS Nano, 2010, 4, 7053-7063.

52 J. F. Quinn, S. J. Pas, A. Quinn, H. P. Yap, R. Suzuki, F. Tuomisto, B. S. Shekibi, J. I. Mardel, A. J. Hill and F. Caruso, J. Am. Chem. Soc., 2012, 134, 19808-19819.

53 Y. Ma, Y. Zhang, B. Wu, W. Sun, Z. Li and J. Sun, Angew. Chem., Int. Ed., 2011, 50, 6254-6257.

54 R. Weissleder, Nat. Biotechnol., 2001, 19, 316-317.

55 H. Kobayashi, M. Ogawa, R. Alford, P. L. Choyke and Y. Urano, Chem. Rev., 2010, 110, 2620-2640.

56 W. M. Leevy, S. T. Gammon, H. Jiang, J. R. Johnson, D. J. Maxwell, E. N. Jackson, M. Marquez, D. Piwnicaworms and B. D. Smith, J. Am. Chem. Soc., 2006, 128, 16476-16477.

57 M. Bauch and J. Dostalek, Opt. Express, 2013, 21, 20470-20483.

58 H. Yuan, J. Liu, Y. Lu, Z. Wang, G. Wei, T. Wu, G. Ye, J. Chen, S. Zhang and X. Zhang, Anal. Chem., 2017, 89, 1045-1048.

59 K. Ray, J. Zhang and J. R. Lakowicz, Anal. Chem., 2008, 80, 7313-7318.

60 A. Izquierdo, S. S. Ono, J. C. Voegel, P. Schaaf and G. Decher, Langmuir, 2005, 21, 7558-7567.

61 D. S. Liu, J. N. Ashcraft, M. M. Mannarino, M. N. Silberstein, A. A. Argun, G. C. Rutledge, M. C. Boyce and P. T. Hammond, Adv. Funct. Mater., 2013, 23, 3087-3095.

62 G. M. Nogueira, D. Banerjee, R. E. Cohen and M. F. Rubner, Langmuir, 2011, 27, 7860-7867.

63 P. C. Lee and D. Meisel, J. Phys. Chem., 1982, 86, 3391-3395.

$64 \mathrm{~J}$. Yguerabide and E. E. Yguerabide, Anal. Biochem., 1998, 262, 137-156.

65 J. R. Lakowicz, Anal. Biochem., 2005, 337, 171-194. 
66 K. L. Kelly, E. Coronado, L. L. Zhao and G. C. Schatz, J. Phys. Chem. B, 2003, 107, 668-677.

67 J. Schmitt, P. Mächtle, D. Eck, H. Möhwald and C. A. Helm, Langmuir, 1999, 15, 3256-3266.

68 P. K. Jain, W. Huang and M. A. El-Sayed, Nano Lett., 2007, 7, 2080-2088.

69 C. Y. Jiang, S. Markutsya and V. V. Tsukruk, Langmuir, 2004, 20, 882-890.

70 G. Schneider and G. Decher, Nano Lett., 2004, 4, 1833- 1839.

71 J. Geng, J. Liang, Y. Wang, G. G. Gurzadyan and B. Liu, J. Phys. Chem. B, 2011, 115, 3281-3288.

72 A. Taflove and S. C. Hagness, Computational Electrodynamics: The Finite-Difference Time-Domain Method, Artech House, 3rd edn, 2005.

73 R. W. Taylor, R. Esteban, S. Mahajan, J. Aizpurua and J. J. Baumberg, J. Phys. Chem. C, 2016, 120, 10512-10522.

74 Y. Zhang, A. Dragan and C. D. Geddes, J. Phys. Chem. C, 2009, 113, 12095-12100.

75 Y. Chen, K. Munechika and D. S. Ginger, Nano Lett., 2007, 7, 690-696.

76 J. Zhang, E. Matveeva, I. Gryczynski, Z. Leonenko and J. R. Lakowicz, J. Phys. Chem. B, 2005, 109, 7969-7975.

77 G. Schneider, G. Decher, N. Nerambourg, R. Praho, M. H. V. Werts and M. Blanchard-Desce, Nano Lett., 2006, 6, 530-536. 